\title{
The incidence of chronic pain following tibial diaphyseal fracture.
}

\author{
Francois Prinsloo $^{1 *}$, Christian Flynn ${ }^{1}$, Matthew Prime ${ }^{2}$, Alex Wickham ${ }^{3}$, Shehan Hettiaratchy ${ }^{4}$ \\ ${ }^{1}$ Department of Surgery, Imperial College London and The University of Sheffield, Sheffield, UK \\ ${ }^{2}$ Department of Orthopaedic Surgery, Imperial College Healthcare NHS Trust, London, UK \\ ${ }^{3}$ Department of Anaesthesia, Imperial College Healthcare NHS Trust, London, UK \\ ${ }^{4}$ Department of Plastic Surgery, Imperial College Healthcare NHS Trust, London, UK
}

\begin{abstract}
Introduction: Chronic pain affects up to $40 \%$ of patients at 7 years after tibial fracture impacting on their quality of life (QoL) and ability to participate in activities of daily living. Major nerves run in close proximity to the tibia and are prone to injury during tibial fracture. Nerve injury can cause acute neuropathic pain, which responds poorly to commonly prescribed analgesics and predisposes patients to developing chronic pain. This study aims to describe the incidence and impact of pain at 6-12 months after tibial fractures.

Methods: Patients admitted to a major trauma centre between 01/01/2016 - 31/12/2016 with isolated tibial fractures were identified using prospectively recorded database eTrauma. Injuries were categorized using the AO classification. Pain and Quality of Life at 6-12 months post injury were assessed using the EuroQol (EQ-5D-5L) questionnaire and the Douleur Neuropathique 4 questionnaire for neuropathic pain.

Results: Forty isolated tibial fractures were identified and 20 were followed up. Pain was reported by $18(90 \%)$ of patients. Ten $(50 \%)$ reported moderate to severe pain, with median pain scores of 2.6/5. Pain scores were significantly greater following high-energy injuries however no significant links were seen between pain and other patient, injury or management factors.

Conclusion: Chronic pain is common following tibial fracture and is largely under-reported. Further prospective multi-center investigation is warranted to better identify those with neuropathic pain and other risk factors for chronic pain. This could guide effective management and improve patient outcomes
\end{abstract}

Keywords: Fracture, Chronic pain, Injury, Trauma.

Accepted on January 21, 2019

\section{Introduction}

Tibial diaphyseal fractures account for $4 \%$ of all fractures [1] and $40 \%$ of all open fractures [2]. They are the commonest long bone fracture in the UK with an annual incidence of 16.9-22 per 100,000 patient years [3-6]. This injury is mostly seen in young men following high-energy mechanisms such as road traffic collisions (RTC) [1,7] and also in older women where, due to the onset of degenerative changes, low-energy mechanisms predominate $[3,4,8]$.

The morphology of tibial fractures is determined by the causative mechanism and can be classified according to the Müller AO classification system [9]. Open fractures make up 23\% of tibial fractures and are commonly classified using the Gustillo and Anderson (GA) system [10]. Closed tibial diaphyseal fractures with good alignment may be managed conservatively; however the majority requires surgical intervention using external and internal fixation techniques [11]. Open fracture management is guided by the BOAST 4 guidelines and should be delivered by a multidisciplinary team [12].

The duration of recovery from tibial fractures is dependent on the injury severity, but frequently rehabilitation can take many months [13] often hindered by chronic pain [14]. Moderate to severe pain persists in $40-78 \%$ of patients 12 months after severe tibial trauma [15-17] and has been reported as present in $40 \%$ of patients 7 years post injury [18]. This complication can leave patients with psychological distress, impacting upon quality of life and impair ability to participate in society $[18,19]$

The aim of this study was to identify the incidence of chronic pain occurring 6 to 12 months post-tibial diaphyseal fracture the hypothesis being that chronic pain will be seen in this patient cohort. Secondary aims were to describe the epidemiology, morphology and acute management of such tibial diaphyseal fractures. These findings can be used to inform the feasibility of future studies.

\section{Methods}

Patients presenting to a London Major Trauma Centre between 01/01/2016-31/12/2016 with fractures of the tibial diaphysis were retrospectively identified using 'eTrauma' (OpenMedical, London, UK), a digital trauma management system. Cases were validated against the hospitals Electronic Health Record (Cerner Corporation, North Kansas City, USA) and submissions to the Trauma Audit and Research Network (TARN) database. Data collection was done at $\mathrm{St}$ Mary's hospital 01/04/2017-30/06/2017. Patient demographic information, injury mechanism, fracture morphology, pain 
score, pain management in the first 24 hours and orthopaedic management were recorded from prospectively documented at the time of injury on the electronic health record. Patients were excluded for predetermined reasons shown in Figure 1. Injuries were classified using the AO classification system or GA system where appropriate. Numerical acute pain scores were converted to mild, moderate or severe for analysis, with scores of 1-3 out of 10 being mild, 4-6 moderate and 7-10 severe pain.

Patients with isolated tibial diaphyseal fractures who presented between $01 / 05 / 2016$ - 30/10/2016 (6 to 12 months post-injury) were identified and contacted by telephone. In order to minimize selection bias, patients were called a total of four times before exclusion ensuring at least one attempt in the morning between 9:00-12:00, once in the afternoon 12:01-17:00 and once in the evening 17:00-19:00. Ethical approval was gained prior to this and verbal consent was sought from each patient to proceed.

Participants were asked to complete the five level EQ-5D-5L to identify the presence of pain and quality of life (QoL). A time trade-off score (TTO) was calculated using EQ-5D-5L, where 1 is full QoL and 0 equivalent to death(20). Patients were also asked to assess their QoL using the EQVAS score (0-100). Questions were subsequently asked from the Douleur Neuropathique 4 (DN4) scoring system to elicit symptoms of neuropathic pain although a complete score could not be gained as patients did not receive physical examination [20,21]. Functional outcomes scores were collected at 6-12 months postinjury using the EQ-5D-5L. To minimize recall bias, questions were asked exactly as stated on official questionnaires.

Statistical analysis was performed using SPSS (version 24.0.0.0, IBM, Chicago, USA). Data was tested for normality using the Shapiro Wilk test. Kruskal-Wallis and Mann-Whitney U tests were used compare the impacts of patient demographic and injury morphology on levels of chronic pain. Spearman's rank correlation coefficient was used to assess associations between EQ-5D outcomes and QoL.

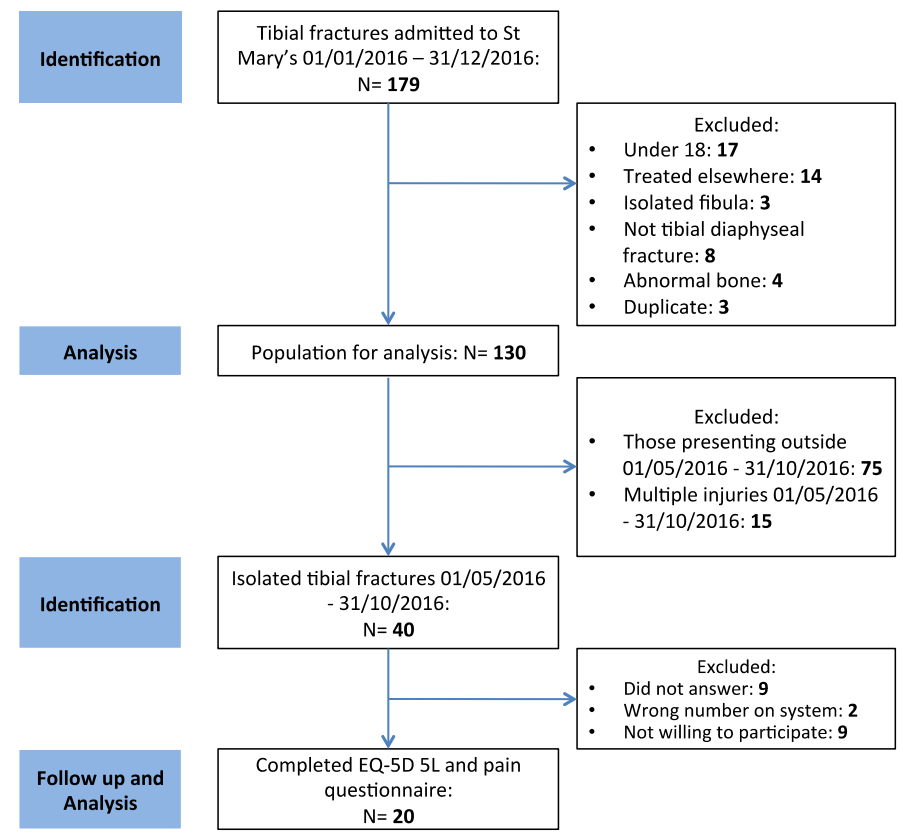

Figure 1. A CONSORT diagram to show the reasons for exclusion in this study.

\section{Results}

This study identified 130 tibial diaphyseal fractures, 93 (71.5\%) of whom were male. The median patient age was 42 years (IQR 31.75-54.25). Men were typically younger, with a median age of 39, compared with 48.5 in women (Figure 1).

\section{Injury mechanisms}

High-energy trauma was responsible for $60.8 \%$ of all injuries and made up $66.7 \%$ of all male and $44.1 \%$ of all female injuries (Figure 2) (Table 1).

\section{Injury morphology}

Isolated injuries accounted for $74.6 \%$ of all fractures. AO type A made up $46.9 \%$, type B $32.3 \%$ and type C $20.8 \%$ as seen in Figure 3.

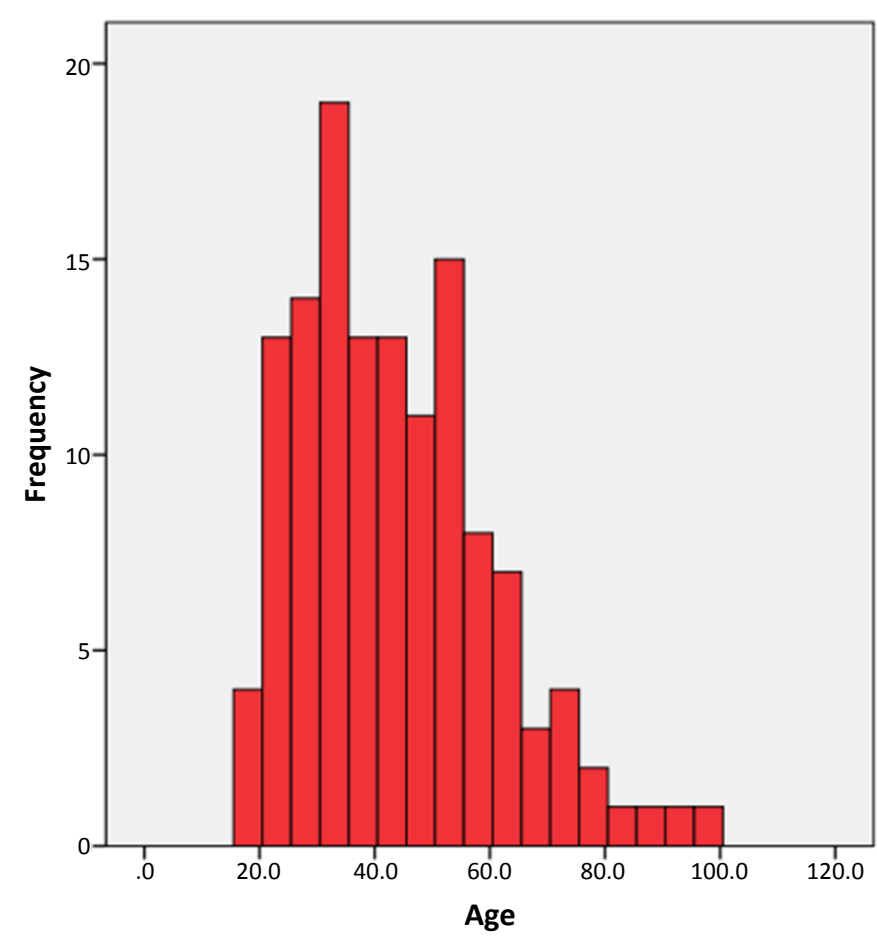

Figure 2. Bar chart showing the age distribution of tibial diaphyseal fractures. Peaks can be observed in those aged 3035 and 50-55.

Table 1. Table showing the breakdown of mechanisms of TF in the overall population.

\begin{tabular}{|l|c|c|c|c|}
\hline Energy & $\begin{array}{c}\text { Mechanism of } \\
\text { injury }\end{array}$ & Male & Female & $\begin{array}{c}\text { Total proportion } \\
\text { of injuries }\end{array}$ \\
\hline High-energy & RTC - Pedestrian & 21 & 12 & $25 \%$ \\
\hline & RTC - Motorbike & 22 & 0 & $17 \%$ \\
\hline & RTC - Cyclist & 2 & 0 & $1 \%$ \\
\hline & RTC - Auto & 1 & 1 & $1 \%$ \\
\hline & Falling object & 5 & 1 & $5 \%$ \\
\hline & Fall from over 2 m & 13 & 1 & $11 \%$ \\
\hline & & & Total high-energy: & $60 \%$ \\
\hline Low-energy & Walking & 8 & 6 & $11 \%$ \\
\hline & Sport & 8 & 0 & $6 \%$ \\
\hline & Fall from under $2 \mathrm{~m}$ & 15 & 13 & $22 \%$ \\
\hline & Assault & 1 & 0 & $1 \%$ \\
\hline & & & Total low-energy: & $40 \%$ \\
\hline
\end{tabular}




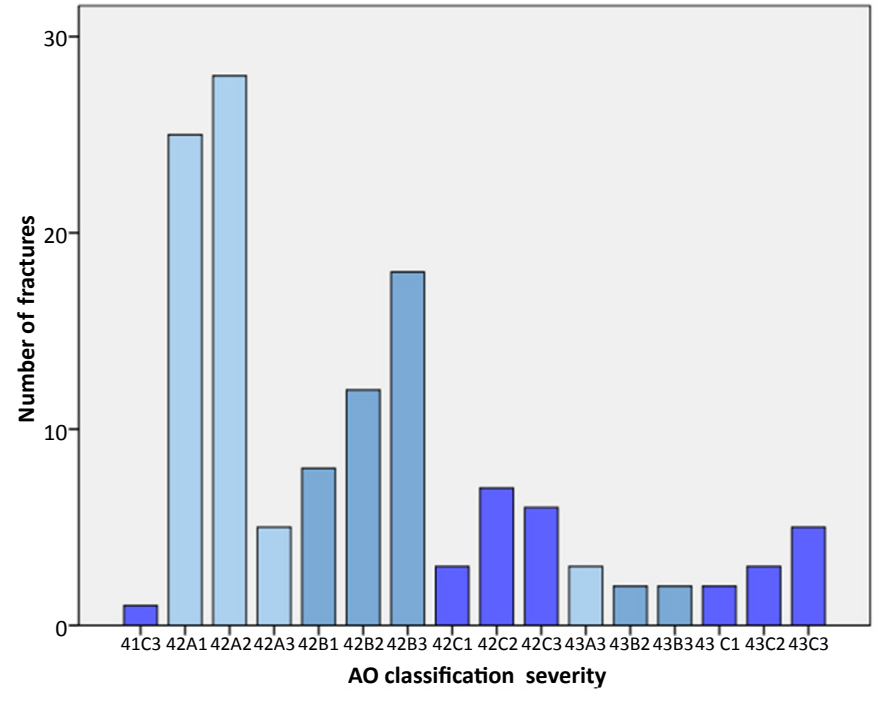

Figure 3. Distribution of fracture severities according to the AO classification system. This includes both open and closed fractures.

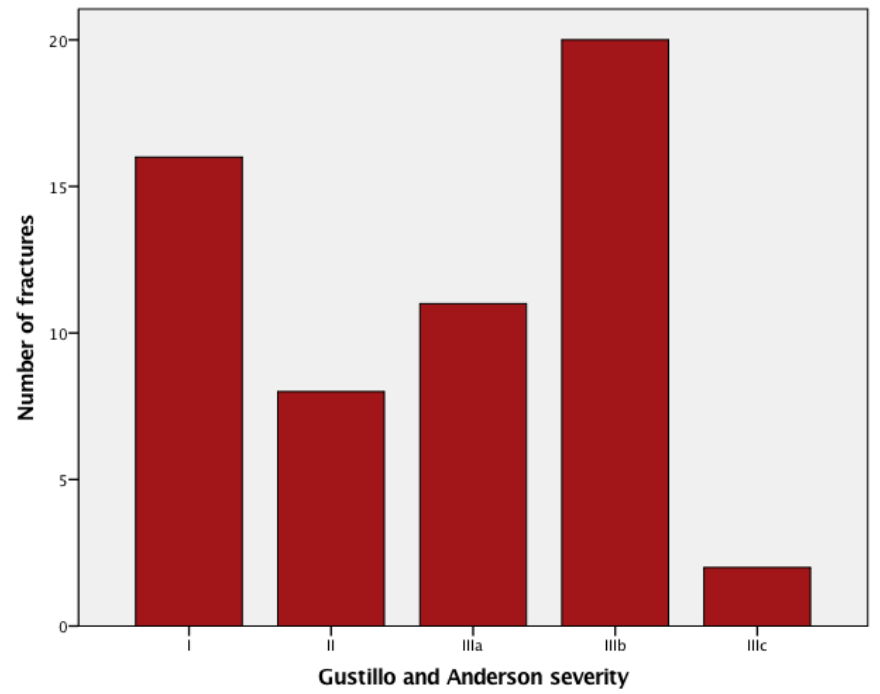

Figure 4. Distribution of open fracture severity according to the GA classification.

Open fractures made up 43\%. GA and AO classifications of severity distribution are shown in Figure 4. High-energy trauma caused $75.4 \%$ of open fractures.

\section{Acute pain and management}

Pain levels in the first 24 hours as recorded in patient notes were 'no pain' in $1(0.8 \%)$, mild in $4(3.1 \%)$, moderate in $28(21.5 \%)$ or severe in $53(40.8 \%)$. Pain scores were not recorded in patient notes for 44 patients (33.8\%). In the first 24 hours, $90 \%$ received paracetamol with an opioid. Additional analgesia with NSAIDs, ketamine or regional blocks using levobupivacaine was given to $12(9.3 \%)$. Neuromodulators were prescribed to 27 patients $(20.8 \%) ; 8$ patients $(29.6 \%)$ of this group received them within 24 hours with a further patients $9(33.3 \%)$ receiving them within a week and $8(29.6 \%)$ between $1-3$ weeks. The remaining 2 patients $(6.9 \%)$ waited over 3 weeks. The median length of neuromodulator prescription was 26 days (IQR 10-154).

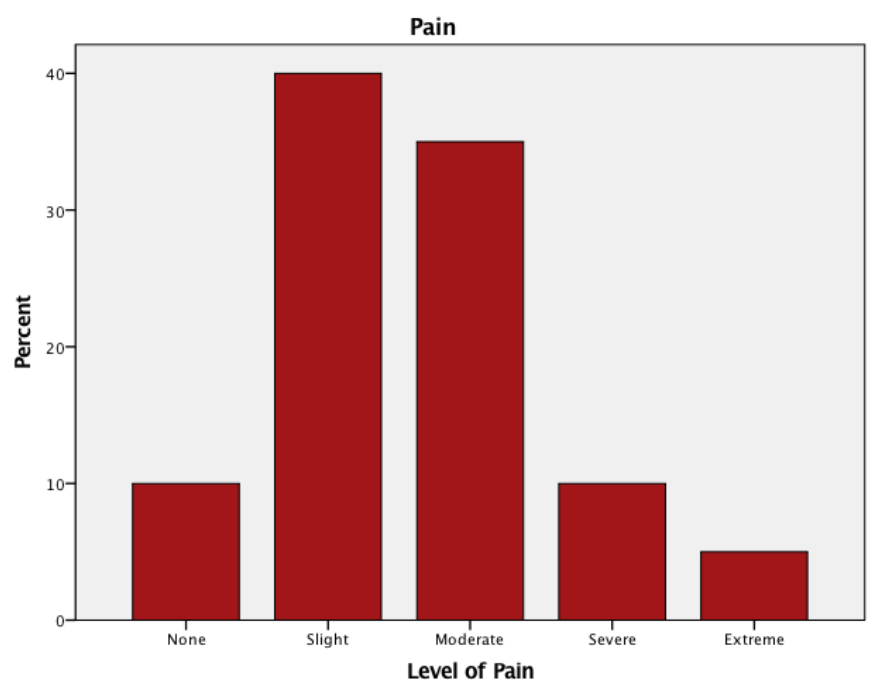

Figure 5. Pain severity reported at 6 to 12 months following TF according to $E Q-5 D-5 L$.

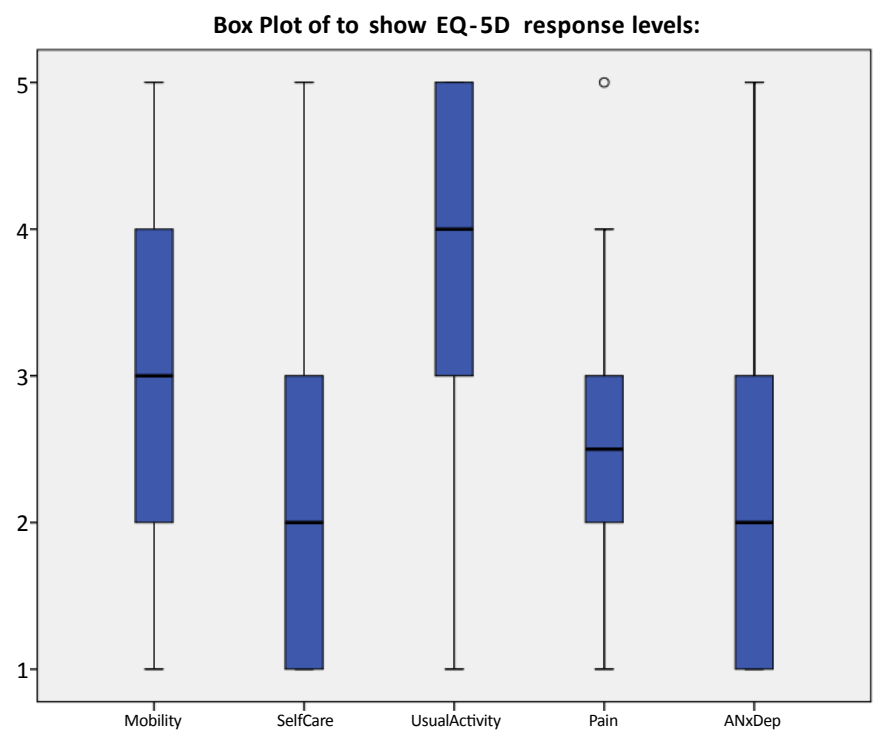

Figure 6. A box and whisker diagram showing patient reported problems according to EQ-5D-5L indices.

\section{Pain at follow up}

Mean follow-up time was 305 days (IQR 244.25-344.25) with a median pain score of 2.5 out of 5 (IQR 2-3). Persisting pain was reported by 18 patients $(90 \%)$ and was graded as moderate to extreme in 10 patients $(50 \%)$, see Figure 5 . Median levels of pain according to EQ5D results were $3 / 5$ in those who sustained high-energy fractures compared with $2 / 5$ for low-energy fractures $(\mathrm{P}=0.016)$. No other patient injury or management related factors significantly impacted on pain at 6-12 months. Features of neuropathic pain were present at follow-up in 7 respondents (35\%). Median pain intensity was 3/5 (IQR 2) in those with neuropathic pain on follow-up according to DN4 compared to $2 / 5$ (IQR 1) in those who did not. Of those with suspected neuropathic pain according to the DN4, 57\% had received neuromodulators. There was no documentation about features of acute neuropathic pain in any patient. Twenty patients did not respond to follow-up; 9 did not answer to any 
of the calls made according to study design; 9 were unwilling to participate; 2 had the wrong contact details recorded.

\section{Quality of life}

EQ-5D responses are shown in Figure 6. Self-reported QoL in this population was perceived as $60.4 \%$ of a full QoL. Calculated QoL was 0.55 , with 1 being full health as calculated using EQ5D TTO scores.

\section{Discussion}

This study found that $90 \%$ of patients report pain persisting 6-12 months after their initial injury, with over half reporting moderate-extreme pain and a third displaying features of neuropathic pain. This is higher than previously reported.

\section{Pain and management}

As in previous literature, acute pain was almost universal for those with pain information recorded at the time of injury. Pain was documented as severe in over half of these. Pain assessment was not recorded in a third of cases, which is inadequate, but in keeping with previous research showing that inpatient pain is both poorly assessed and managed [22]. Acute pain is typically nociceptive, but acute neuropathic pain may also occur following damage to peripheral nerves [23,24]. These major nerves of the lower limb run in close proximity to the tibia making them at particularly high risk of stretch or compression injury and prone to the development of acute neuropathic pain. This results in spontaneous and provoked pain impulses as well as deficits in normal sensation [25].

Poorly controlled acute pain is a risk factor for the development of chronic pain [26] as illustrated by the LEAP study; here acute pain intensity at 3 months following severe tibial fracture was the strongest predictor for pain at 7 years [18].

Chronic pain may occur after tibial fractures for a number of reasons. Cellular and molecular changes at the site of neural injury and in surrounding nerves, including clustering of sodium gated ion channels, expression of $\alpha$-adrenoreceptors, infiltration of inflammatory cells and cytokines and an up regulation of pain transmission receptors leading to peripheral sensitisation [27-29]. Persistent and ectopic action potentials generated from poorly controlled acute pain can result in persistent maladaptive changes in peripheral nerves and the dorsal horn of the spinal cord [27]. Frequent stimulation of the dorsal horn can lead to increased expression and activation of receptors for excitatory neurotransmitters and apoptotic loss of inhibitory GABA neurones [30,31]. Bio-psychosocial variables also contribute to the development of chronic pain, including social group, educational status, low self-efficacy and untreated depression [18,32-36].

Pain persisted at 6-12 months after injury in 90\% of this cohort with half of all patients reporting moderate to extreme pain. This is a greater incidence than previously described and illustrates the extent of the problem of chronic pain following tibial diaphysis fractures. Levels of chronic pain were not found to be affected by surgical management modality, gender, or injury severity. There was however a strong positive correlation between those who sustained high-energy injuries and levels of chronic pain.
This is possibly due to the mechanical strain on peripheral nerves being higher in high-energy injuries thus increasing the likelihood of peripheral nerve damage. This study did not look at the role of fracture union on chronic pain; however this is an important factor to be addresses in future research. A median pain score of $2.5 / 5$ was found which is similar to Rivara et al.'s finding of pain scores of 5.5/10 in patients 1 year after major trauma [35]. Rivara's study focused on more severe Gustillo $\&$ Anderson 3a-3b fractures, whilst our study has noted similar pain scores in less severely injured people. Additionally we found that a third of patients had features of neuropathic pain (according to DN4 diagnostic criteria) at late follow-up and only $50 \%$ of these people had been prescribed neuromodulators, highlighting under-recognition of the problem. Features of acute neuropathic pain were not documented in any patients records.

QoL is greatly reduced in the chronic pain population according to both calculated and self-reported QoL.

Acute pain management of tibial fractures usually involves a combination of paracetamol, non-steroidal anti-inflammatory drugs (NSAIDs) and opioids. This management was seen in $93 \%$ of patients. Neuropathic pain is poorly responsive to these drugs and therefore interest has begun to grow in the use of more advanced treatments such as local anaesthetic nerve blocks, ketamine and neuromodulators such as gabapentin and pregabalin. The neuromodulators are antagonists at the $\alpha 2 \delta$ subunit of presynaptic calcium channels, whose expression is increased in the dorsal horn of patients with chronic pain, and have been shown to be effective for acute neuropathic pain and reduce the risk of chronicity [23,30,37-44]. Thus, better identification of patients who would benefit from such therapy could lead to an improvement in rates of chronic pain in these patients.

\section{Injury mechanisms and morphology}

Whilst the higher incidence in male patients was concordant with previous estimates $[1,3,4]$, the average age of patients was higher and there were fewer sports related injuries than previously reported $[3,4]$. This may be due, in part, to the exclusion of paediatric cases.

High-energy causes, such as RTC's and falls from over 2 metres, predominated. These injuries were almost exclusively sustained by men, whilst women were more likely to suffer low-energy injuries. The high-energy injury mechanisms led to a higher incidence of more serious $\mathrm{AO}$ type $\mathrm{B}$ and $\mathrm{C}$ fractures than previously demonstrated $[1,3]$. Moreover, $43 \%$ of fractures were open, compared to $23.5 \%$ found by Court-Brown and Caesar [4]. These findings are most likely due to the study taking place in a single urban hospital designated as a major trauma centre, where the majority of tibial fractures are caused by RTC's and occupational trauma.

\section{Limitations}

As a single centre study in an urban major trauma centre, some results pertaining to injury cause, injury severity and intervention may only be generalizable to similar centres. Little previous work has focussed on pain after tibial injury meaning it is difficult to adequately power this study to detect the significance 
of chronic pain levels at 6-12 months post injury. Follow-up rates were relatively low, and the patients who declined to participate could represent a population that affected the studies findings in either direction. Due to the nature of trauma, EQ-5D data could not be collected prior to the injury and so it is difficult to see the true effect tibial fracture has on these patients.

\section{Conclusion}

Pain is a significant problem after tibial diaphysis fractures. It is poorly assessed in the acute phase and moderate-severe pain persists in half of patients surveyed at 6-12 months post injury, with observable effects on individuals' quality of life. Education programs highlighting the importance of acute pain management in potential prevention of chronic pain must be developed. Additionally, accurate assessment and documentation of pain is crucial to both improve patient care and facilitate research on the putative link between acute and chronic pain. Further multicenter observational work is need to accurately identify the presence of acute nerve dysfunction, acute and chronic nociceptive and neuropathic pain features and their sequelae in this patient group.

\section{Conflict of Interest and Funding}

There are no conflicts of interest or funding to declare. Project completed as part of a BSc at Imperial College, London.

\section{References}

1. Court-Brown CM, McBirnie J, Tornetta P. The epidemiology of tibial fractures. J Bone Joint Surg Br. 1995;77(3):417-21.

2. Court-Brown CM, Rimmer S, Prakash U, et al. The epidemiology of open long bone fractures. Injury. 1998;29(7):529-34.

3. Larsen P, Elsoe R, Hansen SH, et al. Incidence and epidemiology of tibial shaft fractures. Injury. 2015;46(4):746-50.

4. Court-Brown CM, Bugler KE, Clement ND, et al. The epidemiology of open fractures in adults. A 15-year review. Injury. 2012;43(6):891-7.

5. Weiss RJ, Montgomery SM, Ehlin A, et al. Decreasing incidence of tibial shaft fractures between 1998 and 2004: information based on 10,627 Swedish inpatients. Acta Orthop. 2008;79(4):526-533.

6. Court-Brown CM, Caesar B. Epidemiology of adult fractures: A review. Injury. 2006;37:691-7.

7. Howard M, Court-Brown CM. Epidemiology and management of open fractures of the lower limb. Br J Hosp Med. 1997;57(11):582-587.

8. Singer BR, McLauchlan GJ, Robinson CM, et al. Epidemiology of fractures in 15,000 adults: the influence of age and gender. J Bone Joint Surg Br. 1998;80(2):243-8.

9. Müller ME. Müller AO. Classification of Fractures-Long Bones. AO Foundation. 1987;1-7.

10. Gustilo R, Anderson JT. Prevention of infection in the treatment of one thousand and twenty five open fractures of long bones: retrospective and prospective analyses. J Bone Jt Surg - Ser A. 1976;58(4):453-458.

11. http://www.orthobullets.com/trauma/1045/tibia-shaftfractures

12. https://www.boa.ac.uk/wp-content/uploads/2014/05/ BOAST-4-The-Management-of-Sever-Open-Lower-LimbFractures.pdf

13. Shauver MS, Aravind MS, Chung KC. A qualitative study of recovery from type III-B and III-C tibial fractures. Ann Plast Surg. 2011;66(1):73-9.

14. Perkins ZB, De'Ath HD, Sharp G, et al. Factors affecting outcome after traumatic limb amputation. Br J Surgery. 2012;99:75-86.

15. Dickson DR, Moulder E, Hadland Y, et al. Grade 3 open tibial shaft fractures treated with a circular frame, functional outcome and systematic review of literature. Injury. 2015;46(4):751-758.

16. Hayes C, Browne S, Lantry G, et al. Neuropathic pain in the acute pain service: A prospective survey. Acute Pain. 2002;4(2):45-8.

17. Khan JS, Devereaux PJ, Lemanach Y, et al. Patient coping and expectations about recovery predict the development of chronic post-surgical pain after traumatic tibial fracture repair. Br J Anaesth. 2016;117(3):365-70.

18. Castillo RC, MacKenzie EJ, Wegener ST, et al. Prevalence of chronic pain seven years following limb threatening lower extremity trauma. Pain. 2006;124(3):321-9.

19. McCarthy ML, MacKenzie EJ, Edwin D, et al. Psychological distress associated with severe lower-limb injury. J Bone Joint Surg Am. 2003;85(9):1689-97.

20. Devlin NJ, Shah KK, Feng Y, et al. Valuing health-related quality of life: An EQ-5D-5L value set for England. Health Econ. 2017.

21. Padua L, Briani C, Truini A, et al. Consistence and discrepancy of neuropathic pain screening tools DN4 and ID-Pain. Neurol Sci. 2013;34(3):373-7.

22. Kemp HI, Bantel C, Gordon F, et al. Pain assessment in intensive care (PAINT): an observational study of physician-documented pain assessment in 45 intensive care units in the United Kingdom. Anaesthesia. 2017.

23. Clarke H, Woodhouse LJ, Kennedy D, et al. Strategies aimed at preventing chronic post-surgical pain: Comprehensive perioperative pain management after total joint replacement surgery. Physiother Canada. 2011;63(3):289-304.

24. Merskey H, Bogduk N. IASP task force on taxonomy part III: pain terms, A current list with definitions and notes on usage. IASP Task Force Taxon. 1994;209-14.

25. Schaefer C, Sadosky A, Mann R, et al. Pain severity and the economic burden of neuropathic pain in the United States: BEAT Neuropathic Pain Observational Study. Clin Outcomes Res. 2014;6:483-96. 
26. Costigan M, Scholz J, Woolf CJ. Neuropathic pain: A maladaptive response of the nervous system to damage. Annu Rev Neurosci. 2009;32(1):1-32.

27. Baron R. Mechanisms of disease: neuropathic pain - a clinical perspective. Nat Clin Pract Neurol. 2006;2(2):95-106.

28. Baron R, Levine JD, Fields HL. Cauusalgia and reflex sympathetic dystrophy: Does the sympathetic nervous system contribute to the generation of pain. Muscle Nerve. 1999;22:678-95.

29. Sommer C. Painful neuropathies. Curr Opin Neurol. 2003;16(5):623-8.

30. Dworkin RH, Corbin AE, Young JP, et al. Pregabalin for the treatment of postherpetic neuralgia: a randomized, placebo-controlled trial. Neurol. 2003;60(8):1274-83.

31. Wasner G, Kleinert A, Binder A, et al. Postherpetic neuralgia: Topical lidocaine is effective in nociceptordeprived skin. J Neurol. 2005;252(6):677-86.

32. Portenoy RK, Ugarte C, Fuller I, et al. Population-based survey of pain in the united states: differences among white, african american, and hispanic subjects. J Pain. 2004;5:317-28.

33. Turner JA, Jensen MP, Warms CA, et al. Catastrophizing is associated with pain intensity, psychological distress, and pain-related disability among individuals with chronic pain after spinal cord injury. Pain. 2002;98:127-34.

34. Brittany NR, Sobia K, McCartney C, et al. Systematic review of persistent pain and psychological outcomes following traumatic musculoskeletal injury. J Pain Res. 2013;6:39-51.

35. Rivara FP, Mackenzie EJ, Jurkovich GJ, et al. Prevalence of pain in patients 1 year after major trauma. Arch Surg. 2008;143(3):282-87.

36. Schug SA, Palmer GM, Scott DA, et al. Acute pain management: scientific evidence, fourth edition, 2015. Med J Aust. 2016;204(8):315-17.

37. Mathiesen O, Jacobsen LS, Holm HE, et al. Pregabalin and dexamethasone for postoperative pain control: A randomized controlled study in hip arthroplasty. $\mathrm{Br} \mathrm{J}$ Anaesth. 2008;101(4):535-41.
38. McCartney CJL, Sinha A, Katz J. A qualitative systematic review of the role of $\mathrm{N}$-methyl-D-aspartate receptor antagonists in preventive analgesia. Anesth Analg. 2004;98(5):1385-1400.

39. Cao Y, Wang H, Chiang CY, et al. Pregabalin suppresses nociceptive behavior and central sensitization in a rat trigeminal neuropathic pain model. J Pain. 2013;14(2):193-204.

40. Katz J, Clarke H, Seltzer Z. Preventive analgesia: Quo vadimus? Anesth Analg. 2011;113:1242-53.

41. Saldaña MT, Navarro A, Pérez C, et al. Patient-reportedoutcomes in subjects with painful lumbar or cervical radiculopathy treated with pregabalin: Evidence from medical practice in primary care settings. Rheumatol Int. 2010;30(8):1005-15.

42. Buvanendran A, Kroin JS, Della VCJ, et al. Perioperative oral pregabalin reduces chronic pain after total knee arthroplasty: A prospective, randomized, controlled trial. Anesth Analg. 2010;110(1):199-207.

43. Stacey BR, Dworkin RH, Murphy K, et al. Pregabalin in the treatment of refractory neuropathic pain: Results of a 15-month open-label trial. Pain Med. 2008;9(8):1202-08.

44. Gordh TE, Stubhaug A, Jensen TS, et al. Gabapentin in traumatic nerve injury pain: A randomized, double-blind, placebo-controlled, cross-over, multi-center study. Pain. 2008;138(2):255-66.

\section{*Correspondence to:}

Francois Prinsloo

University of Sheffield

Beech Hill Road

Sheffield

S10 2RX

$\mathrm{UK}$

Tel: 01142225522

E-mail: fdprinsloo1@sheffield.ac.uk 